\title{
The Readiness of Typical Student in Communication By Using Sign Language in Hearing Impairment Integration Programme
}

\author{
Yasin, M. H. M. ${ }^{1}$, Thai, L.K ${ }^{2}$, Tahir, N. T. M. ${ }^{3}$ \\ ${ }^{1,2,3}$ Faculty of Education, The National University of Malaysia, 43600 Bangi, Selangor, Malaysia \\ Corresponding email: mhmy6365@ukm.edu.my
}

\begin{abstract}
This research is regarding the readiness of typical student in communication by using sign language in Hearing Impairment Integration Programme. There were 60 typical students from a Special Education Integration Programme of secondary school in Malacca were chosen as research respondents. The instrument of the research was a set of questionnaire which consisted of four parts, namely Student's demography (Part A), Student's knowledge (Part B), Student's ability to communicate (Part C) and Student's interest to communicate (Part D). The questionnaire was adapted from the research of Asnul Dahar \& Rabiah (2010), namely The Readiness of Students in Following Vocational Subjects at Jerantut District, Rural Secondary School in Pahang. Descriptive analysis was used to analysis the data. Mean score was used to determine the level of respondents' perception of each question. The findings showed a positive relationship between typical students towards communication medium by using sign language. Typical students were seen to be interested in communicating using sign language and were willing to attend the Sign Language class if offered.
\end{abstract}

Keywords: Communication, Integration Programme, Sign Language, Integration, Hearing Impaired Student.

DOI:

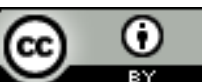

Except where otherwise noted, content on this site is licensed under a Creative Commons Attribution 4.0 International License. 


\section{INTRODUCTION}

Communication is a process of exchange of information and ideas between presenter and recipient. Effective communication can enhance student's selfconcept. According to Zawiyah Mohammad Yusof (2009), the effectiveness of communication refers to the source or information conveyor that can convey information, ideas or knowledge to the recipient accurately and meaningfully without causing confusion or misunderstanding. Meanwhile, according to Abdullah Hassan and Ainon Mohd (2002), the communication during teaching and learning is the process of teacher's experiences sharing with student and vice versa. The Special Education Integration Programme is a programme that uses separate, semi-inclusive and fully inclusive teaching-learning approaches (KPM 2005). Norshidah, Aliza and Zalizan (2009) define Special Education Integration Programme is a programme that provides educational opportunities to student in regular schools. The Integration Programme also refers to the extent to which the inclusion process takes place in education wherein student is integrated, accepted and involved into the school system (Zalizan 2009).

This integration programme plays an important role in facilitating the hearing impaired student in communication process. According to Suarez (2000), one of the objective of implementing this integration programme is to enhance or improve social interaction skills among hearing impaired students. In addition, the programme also helps student to learn better. According to Nunes (2001), the setting up of this integration programme is to access the potential of student in giving impact to academic performance and social adjustments. However, the inability to communicate in the integration programme actually negatively impacts and hinders the success of learning and socialization. Hearing impaired student feels neglected and less be accepted by friends. This led them to isolate and unconfident (Nunes 2001). Therefore, most hearing impaired students show low academic achievement (Gargiulo 2003; Easterbooks \& Baker 2002; Moores 2001). Besides, they often get a low level of standard education (Wilkins \& Hehir, 2008). The study of Zarin Ismail, Safani Bari and Soo Boon Seng (2004) found that social integration among special education student and typical student was at a low level. It means that Special Education Integration Programme still has its' deficiency.

Hence, as whole this study is not only answers the problems related to typical student's communication constraints with hearing impaired student in Special Education Integration Programme (SEIP) of secondary school but also answers the issue of dropping academic performance to school administrators. Therefore, this study is important to ensure that no discrimination on special education programme, and special education student is entitled to obtain an equality of education and unbiased services which suit with their learning needs.

\section{RESEARCH METHODOLOGY}

The method of this study is quantitative. The population of this study are typical students from Special Education Integration Programme (SEIP) of a secondary school in the state of Malacca. Strata sampling is used in research when it is to be 
sure that the subgroups within a selected population will be represented at the same rate or certain rate. The instrument used was a questionnaire containing several parts. All items in the questionnaire are closed-ended question. Five points likert scale questionnaire has been adapted and modified based on the checklist of research done by Asnul Dahar \& Rabbi (2010), namely The Readiness of Students in Following Vocational Subjects at Jerantut District, Rural Secondary School in Pahang. Therefore, a pilot test was done to determine whether the questions given were understood by respondents as well as the requirements of the study. To determine the validity of questionnaire items and to be understood by respondents easily, researcher conducted a pilot study on 10 typical students in an Integration Programme Secondary School. The results were analysed using Cronbach Alpha. The results of the study were shown in the table as below.

\begin{tabular}{|l|c|c|}
\hline \multicolumn{1}{|c|}{ Construct } & $\begin{array}{c}\text { Cronbach's Alpha } \\
\text { Value }\end{array}$ & $\begin{array}{c}\text { The overall value of } \\
\text { Cronbach's Alpha }\end{array}$ \\
\hline Student's knowledge & 0.99 & \multirow{2}{*}{1.10} \\
\cline { 1 - 2 } Student's ability to communicate & 1.15 & \\
\hline Student's interest to communicate & 1.17 & \\
\hline
\end{tabular}

Table 2: Reliability test results

Through the findings of the pilot study, it was found that the overall value of Cronbach's Alpha of questionnaire was 1.10. Therefore, the researcher concludes that the respondent could answers and understands the requirement of the questionnaire. Hence, the set of questionnaires can be used as a guide to the actual study.

Table 2.1: Mean score and interpretation

Table 2.1 shows the interpretation of mean score to assess the level of readiness of typical student in communication by using sign language in hearing impairment of

\begin{tabular}{|c|c|}
\hline Mean Score & Interpretation \\
\hline 1.0 to 2.33 & Low \\
\hline 2.34 to 3.66 & Moderate \\
\hline 3.67 to 5.00 & High \\
\hline
\end{tabular}

Special Education Integration Programme (SEIP). Interpretation of mean score 3.67 to 5.00 indicates the agreement of the item given is at high level, whereas 2.34 to 3.66 shows the agreement of the item given is at moderate level and 1.00 to 2.33 shows the agreement of the item given is at low level.

Hence, questionnaire items have been developed based on the objectives and research questions. The questionnaire is divided into 4 parts, namely Part A, Part B, Part C and Part D. Part A consists of student's demography and educational background that needs to be filled by respondents. Part B, C and D require 
respondent to answer all 30 questions respectively. Items in Part B are intended to identify typical student's knowledge of hearing impaired student and their level of knowledge in sign language. The items in section $\mathrm{C}$ are aimed to identify typical student's ability and Part D student's interest to communicate using sign language. Five Likert Scale are used in the questionnaire, namely strongly disagree (1), disagree (2), uncertain (3), agree (4) and strongly agree (5). It facilitates the respondent to make a right choice for each question. The sample of this study was composed of typical students from Special Education Integration Programme (SEIP) of a secondary school in the state of Malacca. The data were collected through questionnaires and observation. The data analysis was done by using SPSS (Statistical Package for Social Science) version 22 programme.

\section{RESULT AND DISCUSSION}

Part A

Table 3.1: Student’s demography

\begin{tabular}{|l|l|l|}
\hline Gender & Frequency $(f)$ & Percentage (\%) \\
\hline Male & 30 & 50.0 \\
\hline Female & 30 & 50.0 \\
\hline Total & 60 & 100.0 \\
\hline
\end{tabular}

Table 3.1 showed the student's demography such as gender. Respondents were 60 students in term of 30 (50\%) male and 30 (50\%) female.

Table 3.2: Frequency and percentage of the students

\begin{tabular}{|l|l|l|}
\hline Form & Frequency $(f)$ & Percentage (\%) \\
\hline Form 1 & 12 & 20.0 \\
\hline Form 2 & 12 & 20.0 \\
\hline Form 3 & 12 & 20.0 \\
\hline Form 4 & 12 & 20.0 \\
\hline Form 5 & 12 & 20.0 \\
\hline Total & 60 & 100.0 \\
\hline
\end{tabular}

Table 3.2 showed the other item found in part A as Form. Each form was represented by 12 students with percentage of $20.0 \%$ consisting of 6 male and 6 female. 
Part B

\begin{tabular}{|c|c|c|c|c|c|c|c|c|c|}
\hline \multirow[t]{3}{*}{ Item } & \multirow[t]{3}{*}{ Statement } & \multicolumn{5}{|c|}{ Percentage (\%) } & \multirow{3}{*}{$\begin{array}{l}\text { Mea } \\
n\end{array}$} & \multirow{3}{*}{$\begin{array}{l}\text { Std. } \\
\text { Deviation }\end{array}$} & \multirow{3}{*}{$\begin{array}{l}\text { Mean } \\
\text { Score }\end{array}$} \\
\hline & & \multicolumn{5}{|c|}{ Frequency $(f)$} & & & \\
\hline & & SDA & DA & UC & A & SA & & & \\
\hline \multirow[t]{2}{*}{1} & \multirow{2}{*}{$\begin{array}{l}\text { I know } \\
\text { that there } \\
\text { is hearing } \\
\text { impaired } \\
\text { student } \\
\text { class in } \\
\text { my school. }\end{array}$} & 3.3 & 0 & 6.7 & 18.3 & 71.7 & \multirow[t]{2}{*}{4.55} & \multirow[t]{2}{*}{0.89} & \multirow[t]{2}{*}{ High } \\
\hline & & 2 & 0 & 4 & 11 & 43 & & & \\
\hline \multirow[t]{2}{*}{2} & \multirow{2}{*}{$\begin{array}{l}\text { I have } \\
\text { friend of } \\
\text { hearing } \\
\text { impaired. }\end{array}$} & 10.0 & 10.0 & 23.3 & 31.7 & 25.0 & \multirow[t]{2}{*}{3.51} & \multirow[t]{2}{*}{1.25} & \multirow[t]{2}{*}{ Moderate } \\
\hline & & 6 & 6 & 14 & 19 & 15 & & & \\
\hline \multirow[t]{2}{*}{3} & \multirow{2}{*}{$\begin{array}{l}\text { I feel that } \\
\text { the hearing } \\
\text { impaired } \\
\text { friend is } \\
\text { easy to be } \\
\text { friend. }\end{array}$} & 5.0 & 3.3 & 25.0 & 26.7 & 40.0 & \multirow[t]{2}{*}{3.93} & \multirow[t]{2}{*}{1.11} & \multirow[t]{2}{*}{ High } \\
\hline & & 3 & 2 & 15 & 16 & 24 & & & \\
\hline \multirow[t]{2}{*}{4} & \multirow[b]{2}{*}{$\begin{array}{l}\text { I can } \\
\text { understand } \\
\text { the } \\
\text { communic } \\
\text { ation } \\
\text { language } \\
\text { of hearing } \\
\text { impaired } \\
\text { friend in } \\
\text { school. }\end{array}$} & 16.7 & 20.0 & 41.7 & 13.3 & 8.3 & \multirow[t]{2}{*}{2.76} & \multirow[t]{2}{*}{1.14} & \multirow[t]{2}{*}{ Moderate } \\
\hline & & 10 & 12 & 25 & 8 & 5 & & & \\
\hline \multirow[t]{2}{*}{5} & \multirow{2}{*}{$\begin{array}{l}\text { I feel that } \\
\text { a hearing } \\
\text { impaired } \\
\text { friend is as } \\
\text { my other } \\
\text { friends. }\end{array}$} & 8.3 & 8.3 & 30.0 & 28.3 & 25.0 & \multirow[t]{2}{*}{3.53} & \multirow[t]{2}{*}{1.19} & \multirow[t]{2}{*}{ High } \\
\hline & & 5 & 5 & 18 & 17 & 15 & & & \\
\hline
\end{tabular}




\begin{tabular}{|c|c|c|c|c|c|c|c|c|c|}
\hline \multirow[t]{2}{*}{6} & \multirow[b]{2}{*}{$\begin{array}{l}\text { I } \\
\text { understand } \\
\text { that } \\
\text { hearing } \\
\text { impaired } \\
\text { friend is } \\
\text { using sign } \\
\text { language } \\
\text { as their } \\
\text { communic } \\
\text { ation } \\
\text { language. }\end{array}$} & 3.3 & 6.7 & 18.3 & 20.0 & 51.7 & \multirow[t]{2}{*}{4.10} & \multirow[t]{2}{*}{1.13} & \multirow[t]{2}{*}{ High } \\
\hline & & 2 & 4 & 11 & 12 & 31 & & & \\
\hline \multirow[t]{2}{*}{7} & \multirow{2}{*}{$\begin{array}{l}\text { I have } \\
\text { friend with } \\
\text { hearing } \\
\text { impaired } \\
\text { other than } \\
\text { school. }\end{array}$} & 11.7 & 20.0 & 26.7 & 16.7 & 25.0 & \multirow[t]{2}{*}{3.23} & \multirow[t]{2}{*}{1.34} & \multirow[t]{2}{*}{ Moderate } \\
\hline & & 7 & 12 & 16 & 10 & 15 & & & \\
\hline \multirow[t]{2}{*}{8} & \multirow{2}{*}{$\begin{array}{l}\text { I know } \\
\text { hearing } \\
\text { impaired } \\
\text { friend is } \\
\text { having } \\
\text { social } \\
\text { problem. }\end{array}$} & 20.0 & 20.0 & 23.3 & 21.7 & 15.0 & \multirow[t]{2}{*}{2.91} & \multirow[t]{2}{*}{1.35} & \multirow[t]{2}{*}{ Moderate } \\
\hline & & 12 & 12 & 14 & 13 & 9 & & & \\
\hline \multirow[t]{2}{*}{9} & \multirow[b]{2}{*}{$\begin{array}{l}\text { I know the } \\
\text { academic } \\
\text { achieveme } \\
\text { nt of } \\
\text { hearing } \\
\text { impaired } \\
\text { friend is } \\
\text { same as } \\
\text { non- } \\
\text { hearing } \\
\text { impaired } \\
\text { friend. }\end{array}$} & 5.0 & 1.7 & 25.0 & 25.0 & 43.3 & \multirow[t]{2}{*}{4.00} & \multirow[t]{2}{*}{1.10} & \multirow[t]{2}{*}{ High } \\
\hline & & 3 & 1 & 15 & 15 & 26 & & & \\
\hline \multirow[t]{2}{*}{10} & \multirow{2}{*}{$\begin{array}{l}\text { I start to } \\
\text { recognize } \\
\text { people } \\
\text { with } \\
\text { hearing } \\
\text { impaired }\end{array}$} & 15.0 & 10.0 & 13.3 & 18.3 & 43.3 & \multirow[t]{2}{*}{3.65} & \multirow[t]{2}{*}{1.49} & \multirow[t]{2}{*}{ Moderate } \\
\hline & & 9 & 6 & 8 & 11 & 26 & & & \\
\hline
\end{tabular}


in school.

Item 3 showed a high level of mean which 24 respondents (40\%) expressing strong agree (SA) and $26.7 \%$ agree (A) that hearing impaired friend is easy to be friend. Only $5 \%$ with 3 respondents stated that they strongly disagree (SDA) and $3.3 \%$ disagree (DA). Item 5 showed 15 respondents with $25 \%$ at a high level of mean. They were strongly agree (SA) to treat hearing impaired friend as their other typical friend. However, as many as $30 \%$ of 18 respondents were uncertain (UC) that they treat their hearing impaired friend as other typical friend, but only slightly less than $28.3 \%$ respondents agree (A). Next item 9, showing $43.3 \%$ of respondents strongly agree (SA) that academic achievement of hearing impaired friend was same as non-hearing impaired friend. In addition, the percentages of the mean on agree (A) and unsure (UC) were at high level with $25 \%$ respectively. According to indirect observing, hearing impaired students and typical students were competitive to answer teacher's questions during language week at the school assembly. This situation gives a positive perception that hearing impaired student is also learning the same things as other typical student. Item 10 showed the mean at moderate level in such as $43.3 \%$ with 26 respondents start to recognize people with hearing impaired in school, 18.3\% with 11 respondents stated agree (A), 9 respondents (15.0\%) strong disagree (SDA), 6 respondents (10\%) disagreed (DA) and 8 respondents (13.3\%) unsure (UC).

Part C

\begin{tabular}{|c|c|c|c|c|c|c|c|c|c|}
\hline \multirow[t]{3}{*}{ Item } & \multirow[t]{3}{*}{ Statement } & \multicolumn{5}{|c|}{ Percentage (\%) } & \multirow[t]{3}{*}{ Mean } & \multirow{3}{*}{$\begin{array}{l}\text { Std. } \\
\text { Deviation }\end{array}$} & \multirow{3}{*}{$\begin{array}{l}\text { Mean } \\
\text { Score }\end{array}$} \\
\hline & & \multicolumn{5}{|c|}{ Frequency $(f)$} & & & \\
\hline & & DA & A & C & & $\mathbf{A}$ & & & \\
\hline \multirow[t]{2}{*}{1} & \multirow{2}{*}{$\begin{array}{l}\text { I know sign } \\
\text { language. }\end{array}$} & 15.0 & 21.7 & 35.0 & 15.0 & 13.3 & \multirow[t]{2}{*}{2.90} & \multirow[t]{2}{*}{1.23} & \multirow[t]{2}{*}{ Moderate } \\
\hline & & 9 & 13 & 21 & 9 & 8 & & & \\
\hline \multirow[t]{2}{*}{2} & \multirow{2}{*}{$\begin{array}{l}\text { I can } \\
\text { communicate } \\
\text { using sign } \\
\text { language. }\end{array}$} & 25.0 & 30.0 & 20.0 & 11.7 & 13.3 & \multirow[t]{2}{*}{2.58} & \multirow[t]{2}{*}{1.34} & \multirow[t]{2}{*}{ Moderate } \\
\hline & & 15 & 18 & 12 & 7 & 8 & & & \\
\hline \multirow[t]{2}{*}{3} & \multirow{2}{*}{$\begin{array}{l}\text { I used the sign } \\
\text { language with } \\
\text { my hearing } \\
\text { impaired } \\
\text { friend. }\end{array}$} & 13.3 & 36.7 & 21.7 & 15.0 & 13.3 & \multirow[t]{2}{*}{2.78} & \multirow[t]{2}{*}{1.24} & \multirow[t]{2}{*}{ Moderate } \\
\hline & & 8 & 22 & 13 & 9 & 8 & & & \\
\hline 4 & I understand & 18.3 & 26.7 & 25.0 & 18.3 & 11.7 & 2.78 & 1.27 & Moderate \\
\hline
\end{tabular}




\begin{tabular}{|c|c|c|c|c|c|c|c|c|c|}
\hline & $\begin{array}{l}\text { the sign } \\
\text { language used } \\
\text { by hearing } \\
\text { impaired } \\
\text { friend. }\end{array}$ & 11 & 16 & 15 & 11 & 7 & & & \\
\hline \multirow[t]{2}{*}{5} & \multirow{2}{*}{$\begin{array}{l}\text { I often try to } \\
\text { communicate } \\
\text { using sign } \\
\text { language. }\end{array}$} & 10.0 & 20.0 & 18.3 & 33.3 & 18.3 & \multirow[t]{2}{*}{3.30} & \multirow[t]{2}{*}{1.26} & \multirow[t]{2}{*}{ erate } \\
\hline & & 6 & 12 & 11 & 20 & 11 & & & \\
\hline \multirow[t]{2}{*}{6} & \multirow{2}{*}{$\begin{array}{l}\text { I've learned } \\
\text { sign language } \\
\text { with hearing } \\
\text { impaired } \\
\text { student. }\end{array}$} & 18.3 & 26.7 & 15.0 & 20.0 & 20.0 & \multirow[t]{2}{*}{2.96} & \multirow[t]{2}{*}{1.42} & \multirow[t]{2}{*}{ erate ${ }^{\text {Mod }}$} \\
\hline & & 11 & 16 & 9 & 12 & 12 & & & \\
\hline \multirow[t]{2}{*}{7} & \multirow{2}{*}{$\begin{array}{l}\text { Hearing } \\
\text { impaired friend } \\
\text { understands } \\
\text { the sign } \\
\text { language that I } \\
\text { use. }\end{array}$} & 8.3 & 23.3 & 33.3 & 15.0 & 20.0 & \multirow[t]{2}{*}{3.15} & \multirow[t]{2}{*}{1.23} & \multirow[t]{2}{*}{ erate ${ }^{\text {Mod }}$} \\
\hline & & 5 & 14 & 20 & 9 & 12 & & & \\
\hline \multirow[t]{2}{*}{8} & \multirow{2}{*}{$\begin{array}{l}\text { I'm practicing } \\
\text { sign language } \\
\text { with my } \\
\text { classmates. }\end{array}$} & 20.7 & 21.7 & 28.3 & 20.0 & 10.0 & \multirow[t]{2}{*}{2.78} & \multirow[t]{2}{*}{1.26} & \multirow[t]{2}{*}{ erate ${ }^{\text {Mod }}$} \\
\hline & & 12 & 13 & 17 & 12 & 6 & & & \\
\hline \multirow[t]{2}{*}{9} & \multirow{2}{*}{$\begin{array}{l}\text { I am expert in } \\
\text { communicating } \\
\text { using sign } \\
\text { language. }\end{array}$} & 31.7 & 30.0 & 20.0 & 10.0 & 8.3 & \multirow[t]{2}{*}{2.33} & \multirow[t]{2}{*}{1.25} & \multirow[t]{2}{*}{ Low } \\
\hline & & 19 & 18 & 12 & 6 & 5 & & & \\
\hline \multirow[t]{2}{*}{10} & \multirow{2}{*}{$\begin{array}{l}\text { I only know } \\
\text { the basic of } \\
\text { sign language. }\end{array}$} & 15.0 & 15.0 & 25.0 & 25.0 & 20.0 & \multirow[t]{2}{*}{3.20} & \multirow[t]{2}{*}{1.33} & \multirow[t]{2}{*}{ Moderate } \\
\hline & & 9 & 9 & 15 & 15 & 12 & & & \\
\hline
\end{tabular}

Based on data showed as Table 4.5, item 3 stated at moderate level as whole that 22 respondents (36.7\%) disagree (DA) used the sign language with their hearing impaired friend, 9 respondents (15\%) agree (A) and 8 respondents (13.3\%) at strongly agree (SA). The mean of item 5 showed at moderate level that 20 respondents (33.3\%) agreed (A) often try to communicate using sign language whereas 11 respondents (18.3\%) stated uncertain (UC) and strongly agree (SA) in respectively. Through an indirect observation, hearing impaired student did not feel awkward when typical student try to communicate with them. For example, typical student always greet their hearing impaired student during break time by raising up their hands to hearing impaired student as greet sign. Hearing impaired 
student responded to them by raising their hands with a smile too. Such reactions motivate typical student to keep on trying to communicate with hearing impaired student as well as increasing their interest to communicate using sign language. Next, the mean of item 7 is at moderate level as well as 14 respondents (23.3\%) disagree (DA) that hearing impaired friend understands their sign language, 9 respondents (15\%) agree (A) and 12 respondents (20\%) strongly agree (SA). Mean of item 10 also showed at moderate level, which is the number of respondents on uncertain (UC) and agree (A) were same as 12 (25\%) only know the basic of sign language. On the other hand, there were 9 respondents (15\%) and 12 (20\%) respectively on strongly disagree (SDA) and disagree (DA). Besides, typical student came to ask training teacher some of the sign language such as, "name, I, you, handsome, friend, far away, house, and greeting. Even typical student always greet the special education teacher who teaches the hearing impaired student with 'Hello teacher.' This reaction indicates that typical student was so interested in learning and mastering the sign language.

Part D

\begin{tabular}{|c|c|c|c|c|c|c|c|c|c|}
\hline \multirow[t]{3}{*}{ Item } & \multirow[t]{3}{*}{ Statement } & \multicolumn{5}{|c|}{ Percentage (\%) } & \multirow[t]{3}{*}{ Mean } & \multirow{3}{*}{$\begin{array}{l}\text { Std. } \\
\text { Deviation }\end{array}$} & \multirow{3}{*}{$\begin{array}{l}\text { Mean } \\
\text { Score }\end{array}$} \\
\hline & & \multicolumn{5}{|c|}{ Frequency $(f)$} & & & \\
\hline & & SDA & DA & UC & A & SA & & & \\
\hline \multirow[t]{2}{*}{1} & \multirow{2}{*}{$\begin{array}{l}\text { I am interested } \\
\text { in } \\
\text { communicating } \\
\text { using sign } \\
\text { language. }\end{array}$} & 5.0 & 5.0 & 16.7 & 33.3 & 40.0 & \multirow[t]{2}{*}{3.98} & \multirow[t]{2}{*}{1.11} & \multirow[t]{2}{*}{ High } \\
\hline & & 3 & 3 & 10 & 20 & 24 & & & \\
\hline \multirow[t]{2}{*}{2} & \multirow{2}{*}{$\begin{array}{l}\text { I like to } \\
\text { communicate } \\
\text { with hearing } \\
\text { impaired } \\
\text { friend. }\end{array}$} & 3.3 & 5.0 & 21.7 & 40.0 & 30.0 & \multirow[t]{2}{*}{3.88} & \multirow[t]{2}{*}{1.00} & \multirow[t]{2}{*}{ High } \\
\hline & & 2 & 3 & 13 & 24 & 18 & & & \\
\hline \multirow[t]{2}{*}{3} & \multirow{2}{*}{$\begin{array}{l}\text { I agree that } \\
\text { sign language } \\
\text { can be } \\
\text { mastered. }\end{array}$} & 1.7 & 5.0 & 28.3 & 31.7 & 33.3 & \multirow[t]{2}{*}{3.90} & \multirow[t]{2}{*}{0.98} & \multirow[t]{2}{*}{ High } \\
\hline & & 1 & 3 & 17 & 19 & 20 & & & \\
\hline \multirow[t]{2}{*}{4} & \multirow{2}{*}{$\begin{array}{l}\text { Sign language } \\
\text { helps me to } \\
\text { communicate } \\
\text { with hearing }\end{array}$} & 5.0 & 11.7 & 10.0 & 28.3 & 45.0 & \multirow[t]{2}{*}{3.96} & \multirow[t]{2}{*}{1.22} & \multirow[t]{2}{*}{ High } \\
\hline & & 3 & 7 & 6 & 17 & 27 & & & \\
\hline
\end{tabular}




\begin{tabular}{|c|c|c|c|c|c|c|c|c|c|}
\hline & $\begin{array}{l}\text { impaired } \\
\text { student. }\end{array}$ & & & & & & & & \\
\hline \multirow[t]{2}{*}{5} & \multirow{2}{*}{$\begin{array}{l}\text { I think that } \\
\text { sign language } \\
\text { is easy to } \\
\text { master. }\end{array}$} & 3.3 & 5.0 & 23.3 & 45.0 & 23.3 & \multirow[t]{2}{*}{3.80} & \multirow[t]{2}{*}{0.97} & \multirow[t]{2}{*}{ High } \\
\hline & & 2 & 3 & 14 & 27 & 14 & & & \\
\hline \multirow[t]{2}{*}{6} & \multirow{2}{*}{$\begin{array}{l}\text { I feel that sign } \\
\text { language is } \\
\text { important to } \\
\text { learn. }\end{array}$} & 6.7 & 5.0 & 28.3 & 26.7 & 33.3 & \multirow[t]{2}{*}{3.75} & \multirow[t]{2}{*}{1.17} & \multirow[t]{2}{*}{ High } \\
\hline & & 4 & 3 & 17 & 16 & 20 & & & \\
\hline \multirow[t]{2}{*}{7} & \multirow{2}{*}{$\begin{array}{l}\text { I always try to } \\
\text { communicate } \\
\text { with hearing } \\
\text { impaired } \\
\text { friend. }\end{array}$} & 8.3 & 15.0 & 25.0 & 31.7 & 20.0 & \multirow[t]{2}{*}{3.40} & \multirow[t]{2}{*}{1.21} & \multirow[t]{2}{*}{ Moderate } \\
\hline & & 5 & 9 & 15 & 19 & 12 & & & \\
\hline \multirow[t]{2}{*}{8} & \multirow{2}{*}{$\begin{array}{l}\text { I would like to } \\
\text { see hearing } \\
\text { impaired friend } \\
\text { communicate } \\
\text { using sign } \\
\text { language. }\end{array}$} & 6.7 & 8.3 & 10.0 & 26.7 & 48.3 & \multirow[t]{2}{*}{4.01} & \multirow[t]{2}{*}{1.24} & \multirow[t]{2}{*}{ High } \\
\hline & & 4 & 5 & 6 & 16 & 29 & & & \\
\hline \multirow[t]{2}{*}{9} & \multirow{2}{*}{$\begin{array}{l}\text { I agree if there } \\
\text { is } \\
\text { language } \\
\text { condust } \\
\text { school. }\end{array}$} & 8.3 & 3.3 & 8.3 & 21.7 & 58.3 & \multirow[t]{2}{*}{4.18} & \multirow[t]{2}{*}{1.24} & \multirow[t]{2}{*}{ High } \\
\hline & & 5 & 2 & 5 & 13 & 35 & & & \\
\hline \multirow[t]{2}{*}{10} & \multirow{2}{*}{$\begin{array}{l}\text { I would like to } \\
\text { attend the sign } \\
\text { language class. }\end{array}$} & 6.7 & 6.7 & 13.3 & 30.0 & 43.3 & \multirow[t]{2}{*}{3.96} & \multirow[t]{2}{*}{1.20} & \multirow[t]{2}{*}{ High } \\
\hline & & 4 & 4 & 8 & 18 & 26 & & & \\
\hline
\end{tabular}

In Table 4.6.1, item 1 showed a high level of mean. A total of 24 respondents (40\%) strongly agreed (SA) and 20 respondents (33.3\%) agree (A) that they were interested in communicating using sign language. Item 3 stated a high level of mean regarding the student's agreement that sign language can be mastered, which was 33.3\% with 20 respondents strongly agree (SA), 31.7\% with 19 respondents agree (A) and 17 respondents (28.3\%) uncertain (UC). Item 5 also showed high level of the mean. Exactly 45\% (27 respondents) agree (A), 10 respondents (23.3\%) respectively uncertain (UC) and strongly agrees (SA) and 3 respondents (5.0\%) disagree (DA) that sign language is easy to master. 
The mean of item 6 was at high level, with 20 respondents (33.3\%) strongly agree (SA), 16 respondents (26.7\%) agree (A), and 17 respondents (28.3\%) uncertain (UC) that sign language is important to be learned. Item 9 also showed mean at high level in term of agreeing if a sign language class conducts by school with 35 people (58.3\%) strongly agree (SA), 18 people (30\%) agree (A), 4 respondents (6.7\%) for strongly disagree (SDA) and disagree (DA) respectively. The findings also showed that large number of respondents agree if the school conducts a sign language class in school and they wanted to attend the class. Even through an indirect observation, some students ask when the sign language class will begin. The findings of this study stated a very positive reaction from the respondents.

\section{RECOMMENDATIONS}

Some suggestions have been recommended as a step towards improving social communication among students with hearing impairment. Such as school administrator can sets up a class with a simple module for typical student to learn sign language. Typical student can masters the sign language, then problem of communication constraint can be solved easily. Hearing impaired student will be able to move in line with typical student in any circumstance as well as able to elevate school performance. Therefore, integration programme can be seen as move forward in steadily. In order to enhance the commitment of administrator to special education, they need to be given an appropriate explanation and exposure to special education programme. Mohd Najib and Sanisah (2006) found that headmaster and school administrator need to understand the task and gain more exposure about special education because they still lack understanding in the field of special education programme. Therefore, a series of special education courses should be held among school administrators, because the course only be attended by special education frequently without school administrator's participation. Thus, through the courses attended, the school administrators are able to understand deeply on their special education tasks and can strengthen their relationship and commitment with special education programme indirectly.

\section{CONCLUSIONS}

As conclusion, the results of the study indicate typical student is willing and interested in learning, interacting and communicating using sign language. Based on the findings of this study, integration program will be more excellent if we try to understand each other. We can improve the social communication between typical student and hearing impaired student in school. If we have a chance to learn something, definitely an incompatibility will be erased easily. This can be seen as the development of a peaceful and independent nation like Malaysia. If we give others such opportunity to learn our culture and we also learn from each other, certainly misunderstanding or inconsistency can be eliminated. Hence, by giving typical student's opportunity to learn and master sign language will also give an equal opportunity without any discrimination is an effort to enable special education student to be independently and develop their own potential. The best explanation and exposure should also be given to the school administrator so that 
they are to be closer and concerned to the Special Education Integration Programme (SEIP) of secondary school.

\section{REFERENCES}

Hasuria Che Omar dan Rokiah Awang. 2009. Kelestarian Bidang Penterjemahan. Persatuan Penterjemah Malaysia

Syar Meeze Mohd Rashid, Norlidah Alias, Nazean Jomhari, Mohd Yakub Zulkifli Mohd yusof, Zahiah Haris. 2014. Kepentingan bahasa isyarat Malaysia (BIM) dalam pengajaran perkara asas fardhu ain (PAFA) terhadap komuniti pekak. Jurnal Kurikulum \& Pengajaran Asia Pasifik

Muhammad Asif. 2008. Difficulties faced by deaf students and their interaction partners during interaction in inclusive secondary classrooms. Roehampton University.

Mohd Hanafi mohd Yasin, Hasnah Toran, mohd Safani Bari \& Mashitah Khalis. 2012. Perkembangan Akademik dan Sosial Emosi Pelajar Bermasalah Pendengaran. Fakulti Pendidikan, Universsiti Kebangsaan Malaysia.

Azrina Binti Abdul Majid. 2000. Persepsi Guru Khas dan Guru Biasa Terhadap Hubungan Sosialisasi Murid Khas Dengan Murid Normal Di Dalam Program Integrasi. Fakulti Pendidikan. Universiti Kebangsaan Malaysia

Veronica Donald Chin. 2000. Masalah Komunikasi Di Kalangan Pelajar-Pelajar Bermasalah Pendengaran Mengakibatkan Kemerosotan Akademik : Satu Tinjauan Di Sekolah-Sekolah Bermasalah Pendengaran Di Kota Kinabalu Sabah. Fakulti Pendidikan Universiti Kebangsaan Malaysia.

Kamarul Azmi Jasmi. 2012. Kesahan Dan Kebolehpercayaan Dalam Kajian Kualitatif. Fakulti Tamadun Islam. Universiti Teknologi Malaysia

Femi Olivia. 2011. Teknik Ujian Efektif. PT Elex Media Komputindo, Jakarta

Lutfi fauzan. 2009. Peranan Persepsi Dalam Komunikasi. Jurusan Bimbingan Konseling Dan Psikologi. Fakultas Ilmu Pendidikan. Universitas Negeri Malang

Deddy Mulyana. 2010. Ilmu Komunikasi: Suatu Penghantar. Bandung. PT. Remaja Rosdakarya

Mohammad Nawawi. 2012.Pentingnya interaksi Dalam Dunia Pedidikan. Universitas Wiraraja Sumenep.

Nurul Khairun Naim, Mohd Hanafi Mohd Yasin \& Mohd Mokhtar Tahar. Penglibatan Ibu Bapa dalam pendidikan Kanak-kanak Bermasalah Pendengaran. 2014. Fakulti Pendidikan. Universiti Kebangsaan Malaysia.

Mohd Raihan Kasim. Mohd Hanafi Mohd Yasin. \& Mohd Mokhtar Tahar. 2014. Kemudahan Infrastruktur dan Komitmen Pentadbiran Terhadap Program Pendidikan Khas Integrasi Sekolah Rendah. Fakulti Pendidikan. Universiti Kebangsaan Malaysia. 